\title{
Determinanten des Glücks: Lebenszufriedenheit in Europa
}

\begin{abstract}
Subjektives Wohlbefinden ist keine Privatsache: Lebenszufriedenheit ist ungleich verteilt und variiert in jedem Land mit dem Einkommen, dem Beschäftigungsstatus, der Gesundheit und der sozialen Integration. Länderunterschiede bleiben jedoch bestehen. Doch gerade der gesellschaftliche Kontext, wie beispielsweise das jeweilige wohlfahrtsstaatliche System, wurde bislang vernachlässigt, um das Ausmaß an Lebenszufriedenheit über Ländergrenzen hinweg zu erklären. So spiegeln die individuellen Bilanzierungen der Lebenszufriedenheit nicht nur die materielle Lebenslage der Menschen wider, sondern auch ihr Vertrauen in Demokratie, Sozialschutzsysteme und in die Achtung sozialer Rechte.
\end{abstract}

\section{Lebenszufriedenheit als Forschungsthema}

Im Verlauf der Erweiterung der europäischen Union ist das Interesse an Lebensbedingungen und der Verteilung von Lebenschancen in verschiedenen europäischen Ländern erheblich gestiegen. Subjektives Wohlbefinden ist eines von vielen Untersuchungsthemen, das aus dieser ländervergleichenden Perspektive wieder Auftrieb bekommen hat. Lebenszufriedenheit wird dabei als eine Bilanz verstanden, mit der Menschen ihre gegenwärtigen Lebensbedingungen, ihre Möglichkeiten, Präferenzen, Erwartungen und Anpassungsleistungen evaluieren. Lange Zeit verpönt als nicht ernstzunehmende Messinstrumente der Sozialberichterstattung erfahren subjektive Indikatoren derzeit wieder ein hohes Maß an Wertschätzung. Sie dienen, insbesondere bei Ländervergleichen, als Ergänzung zur Messung objektiver Lebensbedingungen wie Einkommen und Bildung. Die Bewertung der Lebensbedingungen aus der Perspektive der Individuen ist mittlerweile als ein entscheidendes Kriterium für Erfolg und Misserfolg von politischem Handeln anerkannt.

Der Beitrag geht mit Hilfe empirischer Illustrationen und Analysen drei Fragestellungen nach:

(1) Unterscheidet sich die Lebenszufriedenheit in den Ländern der europäischen Union, und - falls ja - wie lassen sich diese Unterschiede erklären?
(2) Gibt es länderspezifische Muster zur Erklärung von Lebenszufriedenheit?

(3) Welche Rolle spielen Politik und Kultur als Kontextfaktoren zur Erklärung der Variationen und Determinanten von Lebenszufriedenheit in den einzelnen Ländern?

Von besonderem Interesse ist in unserem Untersuchungszusammenhang der Einfluss des rapiden sozialen Wandels in den postkommunistischen Staaten, in dessen Verlauf Sozialversicherungssysteme zurückgefahren wurden und weite Teile der Bevölkerung Erfahrungen mit Armut und Arbeitslosigkeit machen mussten. Die Unterschiede zwischen alten und neuen EUMitgliedsstaaten hinsichtlich ihrer wirtschaftlichen Prosperität und dem Zugang zu Ressourcen sind beträchtlich. Die Frage ist, in welchem Maße sich diese Unterschiede im subjektiven Wohlbefinden widerspiegeln. Gerade mit Blick auf die heterogenen Lebensbedingungen im erweiterten Europa ist es für die Politik relevant, zu wissen, ob die gleichen Risiken und Benachteiligungen Unzufriedenheit mit sich bringen, selbst wenn sich politische, ökonomische, soziale und kulturelle Rahmenbedingungen von Land zu Land unterscheiden. Für unsere Analysen greifen wir auf den European Quality of Life Survey (EQLS) zurück, der im Jahr 2003 in 28 Ländern erhoben wurde (Alber et al. 2004; Kohler 2007a).

Die Erforschung von Lebenszufriedenheit hat eine langjährige Tradition, und zahlreiche Wissenschaftler unterschiedlicher Disziplinen haben erhebliche Anstrengungen darauf verwendet, Determinanten von Lebenszufriedenheit aus je- weils unterschiedlichen Perspektiven zu beleuchten. Das allgemeine Interesse richtet sich auf den unterschiedlichen Einfluss von biologischen, psychologischen und soziodemografischen Bedingungen, ihre jeweilige Bedeutung sowie ihre Interdependenz.

Die Analyse von Wohlbefinden hat ihren Anfang in umfassenden amerikanischen Studien der 1970er Jahre, in deren Zentrum insbesondere die Rolle von Aspirationen, Werten und Vergleichsgruppen stand. Biologen und Psychologen betonen die Rolle des Individuums und können vergleichsweise starke genetische Einflüsse und Persönlichkeitsmerkmale als Determinanten von Wohlbefinden nachweisen. Sie scheitern allerdings daran, Unterschiede in der Lebenszufriedenheit zwischen Nationen oder Entwicklungen über die Zeit zu erklären (Argyle 1987; Hamer 1996; Inglehart/Klingemann 2000).

Soziologen und Sozialpsychologen hingegen interessieren sich vorrangig für den Einfluss von sozialem Status, Einkommen, Bildung, Arbeitsmarktbedingungen und

Petra Böhnke, Dr., Soziologin am Wissenschaftszentrum Berlin für Sozialforschung (WZB). Arbeitsschwerpunkte: Soziale Ungleichheit und soziale Integration, Wohlfahrtsstaaten im Vergleich. e-mail: boehnke@wzb.eu Ulrich Kohler, Dr., Soziologe am Wissenschaftszentrum Berlin für Sozialforschung (WZB). Arbeitsschwerpunkte: Sozialstrukturanalyse, Politische Soziologie, Methoden der empirischen Sozialforschung e-mail: kohler@wzb.eu 
sozialen Beziehungen auf die Evaluation von Lebensbedingungen (Glatzer/Zapf 1984; Veenhoven 1984; Headey/Wearing 1992). Vor allem Gesundheit, Einkommen und Arbeitslosigkeit sind entscheidende Determinanten, die die Lebenszufriedenheit maßgeblich beeinflussen. Darüber hinaus sind soziale Beziehungen und Ehe Garanten für eine höhere Lebenszufriedenheit (Diener et al. 1999). Allerdings lassen sich mit sozio-demografischen Faktoren nur ca. $20 \%$ der Variation von Lebenszufriedenheit erklären, ein Befund, der in verschiedenen Studien Bestätigung gefunden hat (Campbell et al. 1976; Diener/Suh 1997).

Seit einiger Zeit haben auch die Ökonomen Lebenszufriedenheit als Forschungsthema entdeckt. Sie betrachten subjektives Wohlbefinden in erster Linie als Indikator für den individuellen Nutzen von Lebensbedingungen. Interessante Ergebnisse liegen hier zum Ländervergleich und zum absoluten und relativen Niveau von Lebenszufriedenheit vor (Frey/Stutzer 2002; Oswald 1997).

Es gibt viele Versuche, Determinanten von Wohlbefinden theoretisch zu bestimmen. Sie beginnen bei Aristoteles, gehen über Maslows Bedürfnishierarchie und münden in zahlreichen Indices, die eine lange Reihe von Lebensbereichen aufsummieren, in der Hoffnung, Determinanten von Glück so umfassend wie möglich einzufangen (Hagerty et al. 2001). Erik Allardt (1976; 1993) hat diese Debatte mit der Triade „Having“, „Loving“ und „Being“" zusammengefasst. Dabei bezieht sich „Having“ auf materielle Ressourcen und Lebensbedingungen wie Einkommen, Basiskonsumgüter, Wohnung, Arbeit etc. Die „Loving“Dimension umschreibt die sozialen Bedürfnisse eines Individuums hinsichtlich sozialer Beziehungen, Netzwerke, emotionaler Unterstützung und sozialer Integration im Allgemeinen. „Being“ bezieht sich auf einen allgemeinen Wunsch nach Anerkennung, gesellschaftlicher Integration und Partizipation.

In Anschluss an Arbeiten von Amartya Sen $(1993 ; 1999)$ lässt sich diese letztgenannte Dimension um einen weiteren wichtigen Aspekt ergänzen: Sen betont Verwirklichungschancen (capabilities) als wichtiges Element von Lebensqualität, die Individuen zur Entfaltung elementarer Bedürfnisse und ihrer Selbst benötigen, und unterstreicht damit die Rolle politischer Verantwortlichkeiten und institutioneller

Abb. 1: Lebenszufriedenheit nach UN-Human Development Index

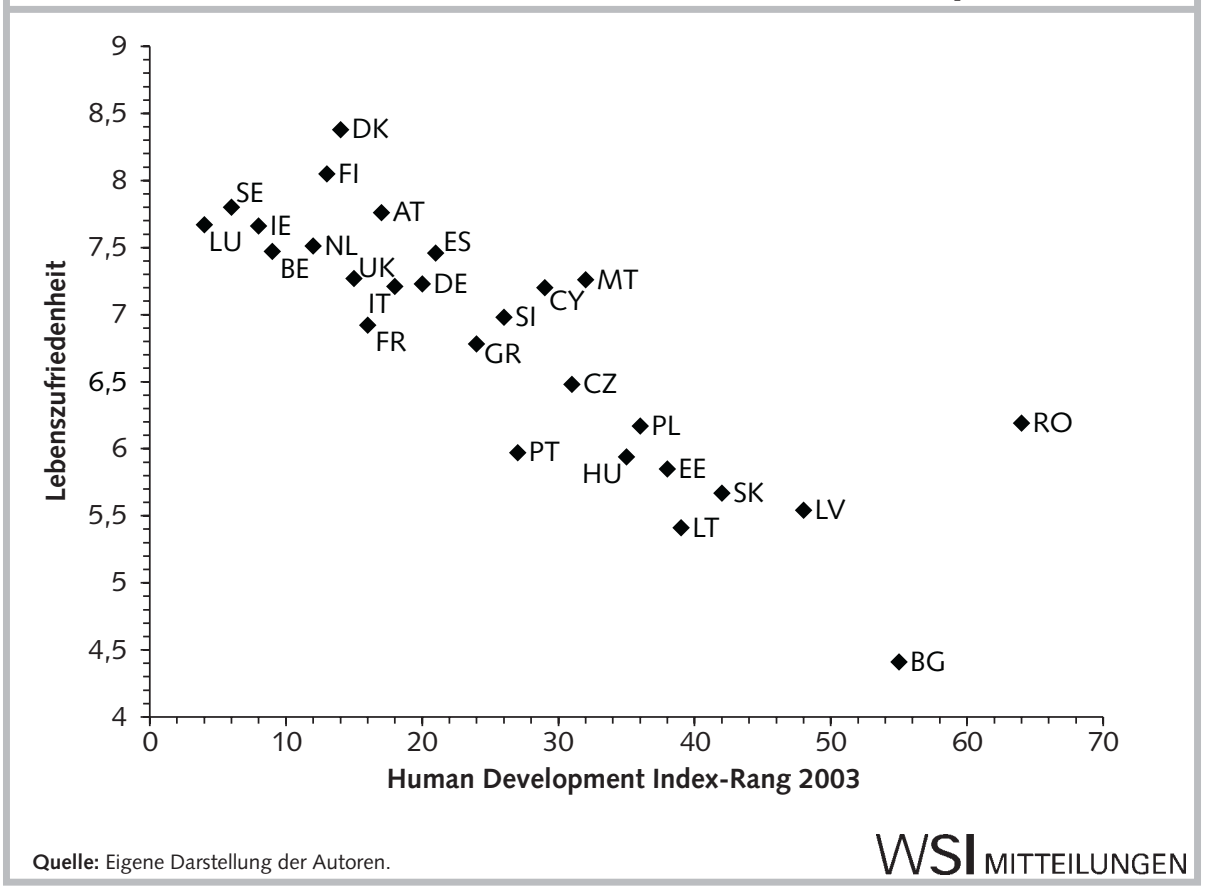

Rahmenbedingungen, die Möglichkeitsspielräume für das Wohlbefinden der Menschen eröffnen bzw. eingrenzen. Für eine ländervergleichende Analyse von Lebenszufriedenheit spielt dieser Gedanke eine besondere Rolle, denn neben möglicherweise universellen individuellen Bedürfnissen für ein glückliches Leben entscheiden länderspezifische Rahmenbedingungen über Einschränkungen und Optionen.

Mit Inglehart lässt sich darüber hinaus auf normative und kulturell verwurzelte Determinanten von Lebenszufriedenheit schließen: Bedürfnisse und Wünsche von Individuen entwickeln sich in einem spezifischen kulturellen und national geprägten Umfeld und müssten sich demzufolge unterscheiden. Eine Folge davon dürfte sein, dass Güter, die universell verfügbar sind, oder Lebensumstände, die unproblematisch zu realisieren sind, weniger erstrebenswert erscheinen, um eine hohe Lebensqualität zu sichern (Inglehart 1977).

\section{Wie zufrieden sind Europäer?}

Für einen ersten Eindruck zeigen wir zunächst, wie Lebenszufriedenheit in Europa verteilt ist. Abbildung 1 weist Länderdurchschnitte für das Jahr 2003 in Verbindung mit dem Entwicklungs- und Mo- dernisierungsstand eines Landes aus, gemessen als Human Development Index (UNDP 2005). Auf diese Weise lässt sich die Heterogenität der Lebensbedingungen und ihr Zusammenspiel mit subjektivem Wohlbefinden eindrücklich darstellen. Die Variationsbreite ist groß: Am zufriedensten ist die dänische Bevölkerung mit ihren Lebensbedingungen. Bulgaren hingegen sind weit abgeschlagen und ziehen eine vergleichsweise düstere Bilanz. Deutlich erkennbar ist überdies die Lücke zwischen alten und neuen Mitgliedsstaaten. In den skandinavischen und kontinentaleuropäischen Ländern ist die Lebenszufriedenheit am höchsten, wohingegen insbesondere die Bevölkerung in den baltischen Staaten und den der EU erst kürzlich beigetretenen Ländern Bulgarien und Rumänien ein deutlich niedrigeres Niveau an subjektivem Wohlbefinden aufweist. Zypern, Malta und Slowenien mischen sich als neue Mitgliedsstaaten unter die alten EU-15-Länder. Auf der anderen Seite finden wir Griechenland und Portugal, deren Lebenszufriedenheitsniveau nicht weit entfernt ist von dem einiger neuer EU-Mitglieder (Abbildung 1).

Mit dem gezeigten deutlichen Zusammenhang von Lebenszufriedenheit und Entwicklungsstand eines Landes reproduzieren wir einen bekannten Befund aus der Literatur: Die höchste Erklärungskraft für Länderunterschiede im subjektiven Wohlbefinden geht vom Niveau ökonomischer Prosperität und Wohlfahrt aus (Ingle- 


\section{Abb. 2: Lebenszufriedenheit nach Einkommen und Pro-Kopf- Bruttoinlandsprodukt}

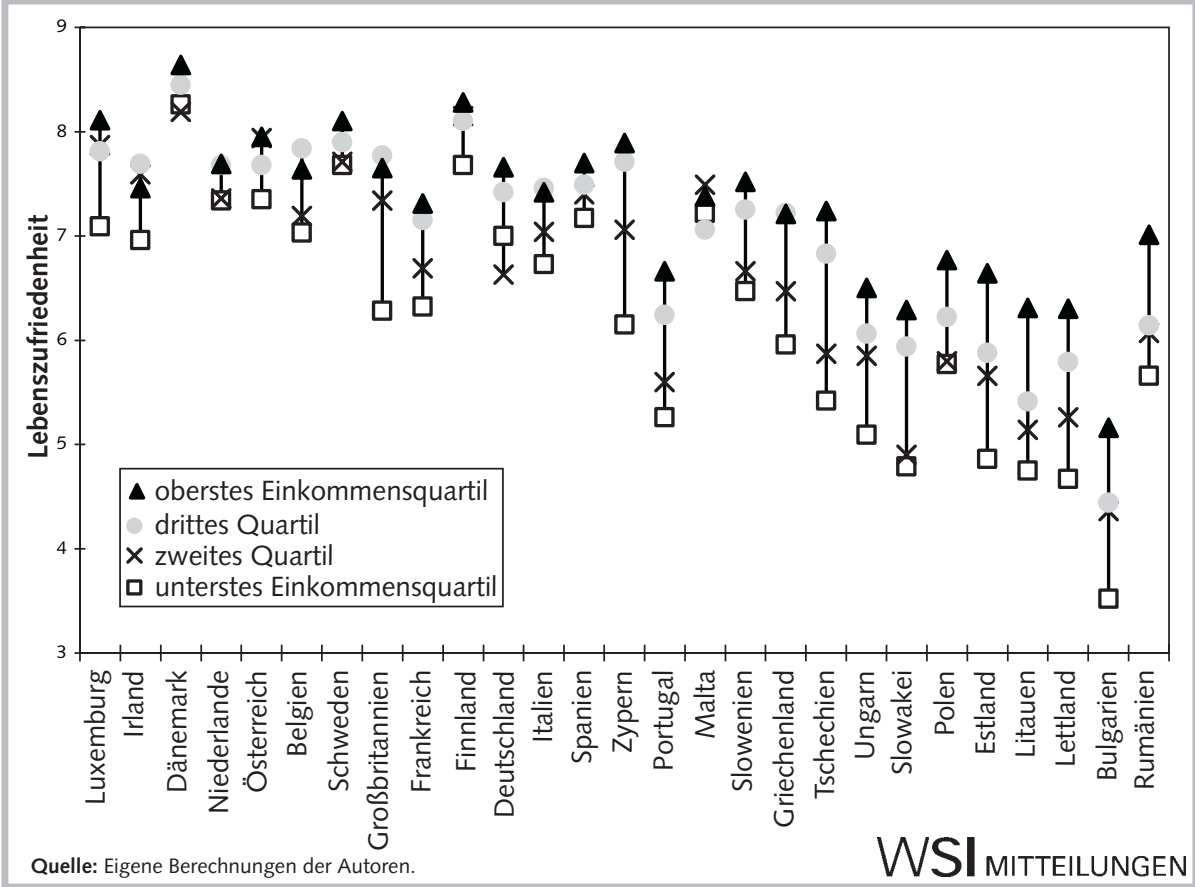

hart/Klingemann 2000; DiTella et al. 2003; Fahey/Smyth 2004). Doch hinter dieser einfachen Beziehung verstecken sich möglicherweise weitere Faktoren: Reiche Länder haben tendenziell stabilere Demokratien, weniger Korruption, bessere soziale Standards und Versorgungssysteme sowie größere Achtung vor sozialen und Menschenrechten. Auf diese kulturellen und politischen Faktoren kommen wir weiter unten zu sprechen. Im nächsten Abschnitt widmen wir uns zunächst länderspezifischen Risikofaktoren, die das Gesamtniveau der Lebenszufriedenheit einer Bevölkerung maßgeblich bestimmen: Wenn das subjektive Wohlbefinden stark durch die persönliche Lebenssituation bestimmt wird, so ist die bevölkerungsweite Lebenszufriedenheit auch eine Folge der nationalspezifischen Verteilung individueller Lebenslagen.

\section{Lebenszufriedenheit und soziale Ungleichheit}

Wir betrachten die Dimensionen sozialer Ungleichheit und ihren Einfluss auf die Lebenszufriedenheit innerhalb eines Landes. Lebenschancen der Individuen unterscheiden sich z. B. nach Einkommen, Gesundheit, Bildungsniveau oder Beschäftigungs- status. Die Frage ist, ob diese Lebensbedingungen Auswirkungen auf das subjektive Wohlbefinden der Menschen haben oder ob Lebenszufriedenheit weitgehend unabhängig von sozialer Ungleichheit ist.

Abbildung 2 zeigt zunächst Einkommensgruppen und ihre Lebenszufriedenheit für europäische Länder (sortiert nach dem Pro-Kopf-Bruttoinlandsprodukt). Zwei wichtige Aussagen lassen sich daran ablesen: Zunächst ist klar erkennbar, dass sich Lebenszufriedenheit in Abhängigkeit des individuellen Einkommens bestimmt. Personen in der Gruppe mit den höchsten Einkommen sind zufriedener als Personen in anderen Einkommensgruppen. Niedrigeinkommensbezieher sind am unzufriedensten mit ihrem Leben.

Darüber hinaus sehen wir ein weiteres Mal, dass das Niveau der Lebenszufriedenheit von der ökonomischen Prosperität eines Landes abhängt. In jeder Einkommensgruppe ist die Bevölkerung reicher Länder zufriedener als die in armen Ländern. In letzter Konsequenz bedeutet dies, dass geringes Einkommen in wohlhabenden EU-15-Staaten mit einer höheren Lebenszufriedenheit einhergehen kann als hohe Einkommen in ärmeren Ländern. Lebenszufriedenheit ist demnach sowohl von der individuellen Position relativ zum nationalen Wohlstandsgefüge als auch von der absoluten Wohlstandsposition eines Landes abhängig (Fahey 2007; Kohler 2007b). Darüber hinaus wissen wir, dass der Einkommenseffekt nicht linear ist, d.h. er ist stärker bei geringen Einkommen und schwächt sich ab, je mehr das Einkommen steigt (Argyle 1999).

Doch nicht nur das verfügbare Einkommen entscheidet maßgeblich über die Lebenszufriedenheit. Dahinter verbergen sich unterschiedliche Bildungsgrade der Befragten und Positionen am Arbeitsmarkt. In einem Regressionsmodell haben wir verschiedene Determinanten in ihrem Einfluss auf die Lebenszufriedenheit getestet. Aus Platzgründen sind die Ergebnisse lediglich für fünf Ländergruppen dargestellt, entsprechen aber den Befunden für die einzelnen Länder: Geringe Bildung, Arbeitslosigkeit und Armut drücken das subjektive Wohlbefinden in allen Ländern massiv. Arbeitslosigkeit ist insbesondere in den kontinentaleuropäischen Ländern ein bedeutsamer Faktor für geringe Lebenszufriedenheit. Dabei spielen nicht nur die mit einem Jobverlust einhergehenden Einkommenseinbußen eine Rolle, sondern auch die an eine Arbeit geknüpfte soziale Integration und das Prestige.

Auch unser Modell zeigt, dass nicht nur die Verfügbarkeit von Ressourcen entscheidend ist. Unabhängig davon ist auch die soziale Integration der Befragten eine wichtige Quelle von Lebenszufriedenheit. Wenn Personen in Notfällen auf Unterstützung aus dem Freundes- und Familienkreis verzichten müssen oder wenige Kontakte mit Freunden und Nachbarn haben, sinkt ihre Lebenszufriedenheit signifikant. Darüber hinaus bestätigen auch diese Modelle, dass Gesundheit länderübergreifend eine bedeutende Determinante von Wohlbefinden ist.

Weitere Berechnungen haben ergeben, dass der Einfluss des Alters auf die Lebenszufriedenheit zwischen den Ländern erheblich variiert. Ältere Menschen sind insbesondere in den postkommunistischen und mediterranen Ländern unzufriedener als die jüngeren, während die älteren Jahrgänge in Nord- und Kontinentaleuropa die zufriedensten sind. Wir haben einige Mechanismen und Kontexteffekte geprüft, um zu ersten Erklärungsversuchen für diese Länderunterschiede zu kommen. Vor allem das Einkommen und die Gesundheit prägen als Individualvariablen die Lebenszufriedenheit. Aber: Sozialstaatliche Regelungen moderieren den Alterseffekt. Die Wirkung von Krankheit und des Ausscheidens aus dem Beruf im Alter auf die Lebenszu- 
friedenheit hängt entscheidend davon ab, wie die entsprechenden Sozialversicherungssysteme im jeweiligen Land gestaltet sind. Hohe Lohnersatzleistungen und eine gute Gesundheitsfürsorge in einem Land schwächen die Rolle von Einkommen und Krankheit als Wirkungsdimension für die Lebenszufriedenheit Älterer ab. In postkommunistischen und mediterranen Staaten, deren Ausgaben für die Alterssicherung und das Gesundheitswesen geringer sind, werden diese typischen Altersfolgen nicht durch sozialstaatliche Regelungen gebremst. Hier führen das geringe Einkommen und das eingeschränkte gesundheitliche Wohlbefinden dazu, dass Ältere weniger zufrieden mit ihrem Leben sind (Böhnke/Kohler 2007).

\section{1 \\ Lebenszufriedenheit und Gesellschaft}

Individuelle Lebensumstände können das Ausmaß an Lebenszufriedenheit nur zum Teil erklären. Aus den vorangegangenen Ausführungen wurde schon deutlich, dass auch kulturelle, ökonomische und politische Rahmenbedingungen, die die Lebenschancen prägen, nicht ohne Einfluss auf das subjektive Wohlbefinden bleiben.

Zum einen unterscheidet sich die Wichtigkeit der Determinanten für Lebenszufriedenheit von Land zu Land in Abhängigkeit gesellschaftspolitischer Rahmenbedingungen. Führt eine Unterausstattung dazu, dass den entsprechenden Gütern ein hoher Stellenwert beigemessen wird? Oder herrschen Anpassungstendenzen vor, sodass insbesondere die Güter bedeutsam werden, die auch zugänglich sind, und Mangel ignoriert wird? Die Befunde bestätigen die Modernisierungstheorie von Inglehart (1977), die besagt, dass leicht zugängliche und weit verbreitete Güter weniger erstrebenswert für ein zufriedenes Leben erscheinen. Lebensstandard und $\mathrm{Zu}$ gang zu materiellen Ressourcen haben insbesondere in den Transformationsländern großen Einfluss auf die Lebenszufriedenheit. In den alten Mitgliedsstaaten spielt der Lebensstandard zwar auch eine zentrale Rolle, aber hier sind gleichzeitig Charakteristika sozialer Integration, wie Familie, soziale Netzwerke und politische Partizipation, wichtig (Delhey 2004; Böhnke 2005). Erstaunlich ist der hohe Stellenwert, den

Tabelle 1: Determinanten von Lebenszufriedenheit nach Ländergruppen

\begin{tabular}{|c|c|c|c|c|c|}
\hline & $\begin{array}{l}\text { Sozialdemo- } \\
\text { kratisch }\end{array}$ & Kontinental & Liberal & Mediterran & $\begin{array}{l}\text { Postkom- } \\
\text { munistisch }\end{array}$ \\
\hline $\begin{array}{l}\text { Bildung } \\
\text { (Referenzkategorie: hohe Bildung) } \\
\text { Mittel } \\
\text { Niedrig }\end{array}$ & $\begin{array}{l}-.018 \\
-.169 *\end{array}$ & $\begin{array}{l}-.240^{*} \\
-.350^{*}\end{array}$ & $\begin{array}{l}-.377 \\
-.179\end{array}$ & $\begin{array}{l}-.121 \\
-.383 *\end{array}$ & $\begin{array}{l}-.256^{*} \\
-.303^{*}\end{array}$ \\
\hline $\begin{array}{l}\text { Erwerbsstatus } \\
\text { (Referenzkategorie: erwerbstätig) } \\
\text { Arbeitslos } \\
\text { Armut }\end{array}$ & $\begin{array}{r}-.395^{*} \\
-1.170^{*}\end{array}$ & $\begin{array}{l}-1.041^{*} \\
-1.481^{*}\end{array}$ & $\begin{array}{r}-.317^{*} \\
-1.560^{*}\end{array}$ & $\begin{array}{r}-.699^{*} \\
-1.229^{*}\end{array}$ & $\begin{array}{r}-.745^{*} \\
-1.262^{*}\end{array}$ \\
\hline $\begin{array}{l}\text { Familienstand } \\
\text { (Referenzkategorie: alleinlebend) } \\
\text { Zusammenlebend } \\
\text { Keine Unterstützung in Notfällen } \\
\text { Wenig Kontakt zu Freunden/Nachbarn } \\
\text { Gesundheitszustand }\end{array}$ & $\begin{array}{r}.477^{*} \\
-.332^{*} \\
-.435^{*}\end{array}$ & $\begin{array}{r}.401^{*} \\
-.297^{*} \\
-.434^{*}\end{array}$ & $\begin{array}{r}.592^{*} \\
-.200^{*} \\
-.687^{*}\end{array}$ & $\begin{array}{r}.375^{*} \\
-.384^{*} \\
-.403^{*}\end{array}$ & $\begin{array}{r}.299^{*} \\
-.567^{*} \\
-.326^{*}\end{array}$ \\
\hline $\begin{array}{l}\text { (Referenzkategorie: krank) } \\
\text { Gesund } \\
\text { Erklärte Varianz } \\
\text { (ohne Länderdummies) - in \% - }\end{array}$ & $\begin{array}{l}.483^{*} \\
13 \%\end{array}$ & $\begin{array}{l}.470^{*} \\
13 \%\end{array}$ & $\begin{array}{r}.357^{*} \\
17 \% \\
\end{array}$ & $\begin{array}{r}.679 * \\
17 \% \\
\end{array}$ & $\begin{array}{c}.576^{*} \\
21 \%\end{array}$ \\
\hline $\begin{array}{l}\text { Lineare Regressionsmodelle, abhängige Variable: } \\
\text { Klammern, ausgewiesen sind B-Koeffizienten, di } \\
\text { des Einflusses einer unabhängigen Variable innerl } \\
\text { Geschlecht und Länder (hier nicht ausgewiesen), } \\
\text { Niederlande, Schweden; Kontinental: Osterreich,, } \\
\text { Irland; Mediterran: Zypern, Griechenland, Malta, } \\
\text { Ungarn, Lettland, Litauen, Polen, Rumänien, Slov } \\
\text { Quelle: EQLS 2003. }\end{array}$ & $\begin{array}{l}\text { Lebenszufriedenh } \\
\text { e zwischen den Lär } \\
\text { halb eines Landes } \\
\text { * statistisch signifi } \\
\text { Belgien, Frankreic } \\
\text { Spanien, Portugal } \\
\text { wakei, Slowenien. }\end{array}$ & $\begin{array}{l}\text {, Referenzk } \\
\text { ergruppen v } \\
\text { ssagen; die } \\
\text { nt mit } p<= \\
\text { Deutschlan } \\
\text { ostkommur }\end{array}$ & $\begin{array}{l}\text { rien für } \\
\text { chbar si } \\
\text { :lle sind } \\
\text { Sozialc } \\
\text { ien, Lux } \\
\text { h: Bulga }\end{array}$ & $\begin{array}{l}\text { hängige Varia } \\
\text { per nichts übe } \\
\text { zlich kontrollie } \\
\text { kratisch: Däne } \\
\text { arg; Liberal: G } \\
\text { Tschechische } \\
\text { MIT }\end{array}$ & $\begin{array}{l}\text { en in } \\
\text { die Stärke } \\
\text { t für Alter, } \\
\text { nark, Finnland, } \\
\text { Bbritannien, } \\
\text { epublik, Estland, } \\
\text { EILUNGEN }\end{array}$ \\
\hline
\end{tabular}

die Bewertung der Gesellschaft für die individuelle Lebenszufriedenheit hat. In allen Ländern sind Menschen bedeutend unzufriedener, die wenig Vertrauen in die Sozialversicherungssysteme wie auch in andere Menschen haben, viele Konflikte zwischen gesellschaftlichen Gruppen wahrnehmen und enttäuscht über das Gesundheits- und Bildungswesen ihres Landes sind. Insbesondere für die Lebenszufriedenheit der Menschen in den Transformationsländern spielt die gesellschaftliche Qualität eine wichtige Rolle: Je niedriger die ökonomische Prosperität eines Landes, je niedriger die Sozialschutzausgaben, die Lebenserwartung oder die Effektivität der Regierung, desto stärker und negativer schlägt die wahrgenommene Qualität der Gesellschaft für die Lebenszufriedenheit zu Buche (Böhnke 2007).

Nur wenige Studien beschäftigen sich mit makrostrukturellen Effekten, um die Variation von Lebenszufriedenheit zwischen den Ländern zu erklären. Die Interaktion zwischen individuellen länderspezifischen Faktoren und gesamtgesellschaftlichen Rahmenbedingungen wird dabei meistens außer Acht gelassen. Neben dem Wohlstandsniveau eines Landes erweisen sich Indikatoren zur Demokratieperformanz als aussagekräftig, um die unterschiedliche Lebenszufriedenheit über Ländergrenzen hinweg zu erklären (Diener/ Suh 1999; Inglehart/Klingemann 2000; Diener/Lucas 2000; Helliwell 2003). Weiterhin sind die Verbreitung von Arbeitslo- sigkeit sowie die Höhe der Arbeitslosenunterstützung (Clark/Oswald 1994; DiTella et al. 2001) entscheidend. Gleiches gilt für kulturelle Faktoren und Wertausprägungen wie etwa das Vorherrschen individualistischer und postmaterialistischer Werte (Hofstede 2001). Haller/Hadler (2006) kommen zu dem Ergebnis, dass Lebenszufriedenheit auf einer Interaktion zwischen individuellen Charakteristika, Aspirationen und makrostrukturellen Rahmenbedingungen beruht.

Im Folgenden wählen wir eine einfache Darstellungsart, um den Zusammenhang von gesellschaftlichen Rahmenbedingungen und individueller Lebenszufriedenheit anschaulich zu machen: Abbildung 3 zeigt den Einfluss von einigen Kontextfaktoren auf die hinsichtlich Geschlecht, Bildung, Einkommen, Familienstand und Alter standardisierte Lebenszufriedenheit. Variationen der Lebenszufriedenheit zwischen den Ländern können in diesen strukturell standardisierten Lebenszufriedenheiten nicht auf Unterschiede in der Verteilung soziodemografischer Merkmale zurückgeführt werden. Die Ursache für diese Unterschiede muss darum in anderen gesellschaftlichen Rahmenbedingungen liegen.

Die Befunde machen deutlich: Es gibt einen klaren Zusammenhang zwischen der strukturell standardisierten Lebenszufriedenheit und gesellschaftlichen Rahmenbedingungen: Je höher die Sozialschutzausgaben, je effektiver das politische System und je höher die Lebenserwartung, desto 


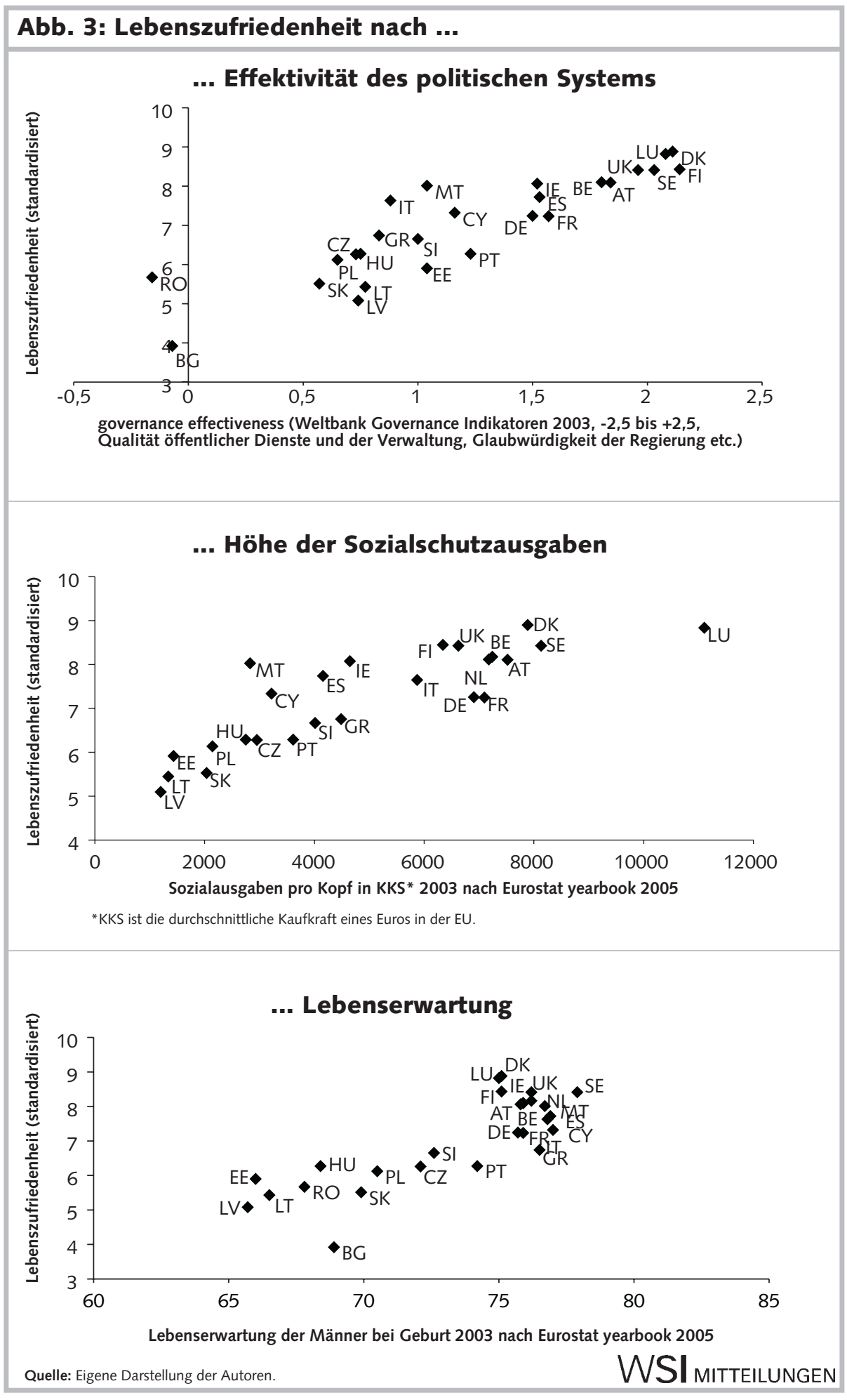

zufriedener sind die Menschen in den einzelnen Ländern mit ihrem Leben. Weitere Kontextfaktoren sind signifikant, wenngleich nicht in dieser Stärke: die Arbeitslosenquote, die Verbreitung extremer Armut, Säuglingssterblichkeit, politische Rechte und Freiheitsgrade. Die Menschen sind also durchaus in der Lage, die Qualität ihrer Gesellschaft einzuschätzen und nehmen sie als Möglichkeitsraum oder Restriktion für nen Landes und ist gleichzeitig ein Gradmesser für die Qualität der politischen und kulturellen Rahmenbedingungen eines Landes im Vergleich zu anderen, ein glückliches Leben $\mathrm{zu}$ ermöglichen. Um das Wohlbefinden der Menschen zu erhöhen, reicht es also nicht aus, soziale Benachteiligung, wie etwa Armut, zu bekämpfen. Die politische und institutionelle Struktur eines Landes ist darüber hinaus ein maßgebliches Kriterium für Lebensqualität. Stabile und verlässliche Demokratien, die Achtung der Menschenrechte und ein universeller Zugang zu Sozialsystemen, die Notlagen abmildern, schaffen Zuversicht und Optionenvielfalt und sind Garanten dafür, dass individuelle Lebensbedingungen positiv wahrgenommen werden.

\section{Resümee}

Die Variationsbreite hinsichtlich der Lebenszufriedenheit europäischer Bürger ist groß und spiegelt die äußerst heterogenen Lebensbedingungen in der erweiterten Union wider. Innerhalb der alten EU-Mitgliedsstaaten ist das Nord-Süd-Gefälle nach wie vor präsent. Portugiesen und Griechen sind unzufriedener mit ihrem Leben als die Bevölkerung in Skandinavien. Davon unterscheidet sich nochmals deutlich das Niveau subjektiven Wohlbefindens in den postkommunistischen neuen Mitgliedsstaaten. Insbesondere die Bevölkerung im Baltikum sowie in Bulgarien und Rumänien ist vergleichsweise unzufrieden mit ihren Lebensbedingungen.

Unabhängig von den Länderunterschieden steht die individuelle Lebenszufriedenheit in einem engen Zusammenhang mit dem Zugang zu materiellen Ressourcen, mit Wohnbedingungen und der Position am Arbeitsmarkt. Armut und Arbeitslosigkeit lassen länderübergreifend die Lebenszufriedenheit sinken. Familienanschluss und emotionale Unterstützung können diese Tendenz abschwächen. Dauerhafte Benachteiligung bedeutet aber oft auch deren Kumulation, und materielle Notlagen führen nicht selten zu Schwierigkeiten im Freundes- und Familienkreis und bringen gesundheitliche Beeinträchtigungen mit sich.

Die große Diskrepanz zwischen der Lebenszufriedenheit von Schweden und Bulgaren lässt sich mit ökonomischen, institu- 
tionellen und gesellschaftspolitischen Rahmenbedingungen gut erklären. Es zeigt sich, dass das Wohlfahrtsniveau und die Struktur der Sozialversicherungssysteme einen deutlichen Einfluss auf subjektives Wohlbefinden ausüben. Wird sozialer Schutz als prekär empfunden und mangelt es an Vertrauen in das politische System, sehen Menschen ihre persönlichen Lebensbedingungen davon beeinträchtigt.
Fazit: Wie zufrieden jemand mit seinem Leben ist, hängt nicht nur von seiner Persönlichkeitsstruktur und privaten Lebensumständen ab. Strukturelle Ursachen und Mechanismen sozialer Ungleichheit prägen maßgeblich das subjektive Wohlbefinden. Darüber hinaus ist es bedeutsam, in welche institutionellen und politischen Kontexte individuelle Lebenswege eingebettet sind. Individuelle Lebensumstände und gesellschaftliche Rahmenbedingungen gehen unabhängig voneinander in die Lebensbilanz eines Individuums ein. Die sehr niedrige Lebenszufriedenheit in vielen osteuropäischen Staaten ist vor allem auf unzureichende soziale Absicherung und mangelndes Vertrauen in das politische System zurückzuführen, was von den Menschen klar als Restriktion ihrer Handlungsoptionen wahrgenommen wird.

\section{LITERATUR}

Alber, J./Delhey, J./Keck, W./Nauenburg, R. (Hrsg.) (2004): Quality of Life in Europe, First European Quality of Life Survey 2003, Luxembourg Allardt, E. (1976): Dimensions of welfare in a comparative Scandinavian Study, in: Acta Sociologica 19, 3, pp. 227-239

Allardt, E. (1993): Having, Loving, Being: An Alternative to the Swedish Model of Welfare Research, in: Nussbaum, M./Sen, A. (Hrsg.): The Quality of Life, Oxford, pp. 88-94

Argyle, M. (1987): The psychology of happiness, London

Argyle, M. (1999): Causes and Correlates of Happiness, in: Kahnemann, D./Diener, E./Schwarz, N. (Eds.): Well-Being: The Foundations of Hedonic Psychology, New York, pp. 353-373

Böhnke, P. (2005): First European Quality of Life Survey: Life Satisfaction, Happiness and Sense of Belonging, Luxembourg

Böhnke, P. (2007): Policy or Privacy - What matters most for individual well beeing? Determinants of life satisfaction in the enlarged Europe. WZB Discussion Paper SP/2007-203, Berlin (im Erscheinen)

Böhnke, P./Kohler, U. (2007): Im richtigen Alter am richtigen Ort? Lebenszufriedenheit in Europa, in: Rehberg, K.-S. (Eds.): Die Natur der Gesellschaft, 33. Kongress der Deutschen Gesellschaft für Soziologie (im Erscheinen)

Campbell, A./Converse, P. E./Rodgers, W. L. (1976): The quality of American life. Perceptions, evaluations, and satisfactions, New York Clark, A. E./Oswald, A. J. (1994): Unhapppiness and Unemployment, in: Economic Journal 104, 424, pp. 648-659

Delhey, J. (2004): Life Satisfaction in the Enlarged Europe, Luxembourg Diener, E./Lucas, R. (2000): Explaining differences in societal levels of happiness: Relative standards, need fulfillment, culture, and evaluation theory, in: Journal of Happiness Studies: An Interdisciplinary Periodical on Subjective Well-Being 1, pp. 41-78

Diener, E./Suh, E. M. (1997): Measuring quality of life: Economic, social, and subjective indicators, in: Social Indicators Research 40, pp. 189-216 Diener, E./Suh, E. M. (1999): National Differences in Subjective WellBeing, in: Kahneman, D./Diener. E./Schwarz, N. (Hrsg.): Well-Being: The Foundations of Hedonic Psychology, New York, pp. 434-450

Diener, E./Suh, E. M./Lucas, R./Smith, H. (1999): Subjective Well-Being: Three Decades of Progress, in: Psychological Bulletin 125, pp. 276-302
Di Tella, R./MacCulloch, R. J./Oswald, A. J. (2001): Preferences over inflation and unemployment: Evidence from surveys of happiness, in: The American Economic Review 91, 1, pp. 335-341

Di Tella, R./MacCulloch, R. J./Oswald, A. J. (2003): The Macroeconomics of happiness, in: Review of Economics and Statistics 85, 4, pp. 809827

Fahey, T./Smyth, E. (2004): Do Subjective Indicators Measure Welfare? Evidence from 33 European societies, in: European Societies 6, 1, pp. 5-27 Fahey, T. (2007): The Case for an EU-wide Measure of Poverty, in: European Sociological Review, 23, pp. 35-47

Frey, B. S./Stutzer, A. (2002): Happiness and economics. How the economy and institutions affect well-being, Princeton Glatzer, W./Zapf, W. (1984) (Hrsg.): Lebensqualität in der Bundesrepublik. Objektive Lebensbedingungen und subjektives Wohlbefinden, Frankfurt/New York

Hagerty, M. R./Cummins, R. A./Ferriss, A. L./Land, K./Michalos, A. C./Peterson, M./Sharpe, A./Sirgy, J./Vogel, J. (2001): Quality of life indexes for national policy: review and agenda for research, in: Social Indicators Research 55, 1, pp. 1-96

Haller, M./Hadler, M. (2006): How social relations and structures can produce happiness and unhappiness: an international comparative analysis, in: Social Indicators Research 75, pp. 169-216

Hamer, D. H. (1996): The Heritability of Happiness, in: Nature Genetics 14, 2, pp. 125-126

Headey, B./Wearing, A. (1992): Understanding happiness. A theory of subjective well-being, Melbourne

Helliwell, J. F. (2003): How's life? Combining individual and national variables to explain subjective well-being, in: Economic Modelling 20, pp. 331-360

Hofstede, G. (2001): Culture's consequences. Comparing values, behaviours, institutions and organisations across nations, Thousand Oaks/ London/New Delhi

Inglehart, R. (1977): The Silent Revolution. Changing Values and Political Styles Among Western Publics, Princeton

Inglehart, R./Klingemann, H.-D. (2000): Genes, culture, democracy, and happiness, in: Diener, E./Suh, E. M. (Hrsg.): Culture and Subjective Wellbeing, Cambridge, pp. 165-183. 
Kohler, U. (2007a): Quality assessment of European Surveys. Towards an open method of coordination for survey data, in: Alber, J./Fahey, T./Saraceno, C. (Eds.): Handbook of quality of life in enlargement Europe (im Erscheinen)

Kohler, U. (2007b): Containers, Europeanisation and individualisation: Empirical implications of general decriptions of society, in: Scherer, S./ Pollack, R./Otte, G./Gangl, M. (Eds.): From Origin to Destination. Trends and Mechanisms in Social Stratification Research. Frankfurt a. M./New York, pp. 292-319
Oswald, A. J. (1997): Happiness and Economic Performance, in: Economic Journal 107, 445, pp. 1815-1831

Sen, A. (1993): Capability and well-being, in Nussbaum, M./Sen, A.: The Quality of life, Oxford, pp. 30-53

Sen, A. (1999): Development as Freedom, Oxford

United Nations Development Programme (UNDP) (2005): Human Development Report 2005, New York

Veenhoven, R. (1984): Conditions of happiness, Dordrecht

\title{
Gleichstellung ohne Vereinbarkeit - Vereinbarkeit ohne Gleichstellung? Zum Verhältnis von Gleichstellungspolitik und Familienpolitik
}

\author{
Veranstaltung des WSI
}

27./28. September 2007 in Berlin

Die Vereinbarkeit von Beruf und Familie ist in den vergangenen Jahren zu einem der wichtigsten sozialpolitischen Themen geworden - für die Europäische Union, deren Europäische Beschäftigungsstrategie auf die Erhöhung der Frauenbeschäftigung ausgerichtet ist, wie auch für die deutsche Bundesregierung, die Frauen als Fachkräfte entdeckt hat und die sinkende Geburtenrate als Bedrohung für den Erhalt der sozialen Sicherungssysteme interpretiert. Ein solcher Handlungsansatz ist also nicht primär gleichstellungspolitisch, sondern vorwiegend ökonomisch motiviert. Wie ist er aus gleichstellungspolitischer Perspektive zu bewerten?

Die Veranstaltung setzt die mit den beiden Vorgängertagungen „Strategien der Gleichstellung von Frauen und Männern in der Privatwirtschaft“ (2005) und „Geschlechterverhältnisse im Betrieb: Gleiche Verhältnisse - gleiche Chancen?“ (2006) begonnene Debatte und wissenschaftliche Analyse fort. Die Tagung 2007 legt ihren Fokus auf das Verhältnis von Familien- und Gleichstellungspolitik und thematisiert flankierend Veränderungen in der Arbeitswelt und in den Haushalten. Zielgruppen der Veranstaltung sind Wissenschaftlerinnen und Wissenschaftler, Politikerinnen und Politiker, Gewerkschafterinnen und Gewerkschafter, Gleichstellungsbeauftragte, Studierende und andere Interessierte.

Es referieren u.a. Karin Gottschall, Karin Jurczyk, Hildegard Nickel, Ulla Björnberg, Helma Lutz und Hildegard Theobald.

Weitere Informationen: www.boeckler.de/veranstaltungen

Anmeldung: Katharina Kruse, Hans-Böckler-Stiftung, e-mail: katharina-kruse@boeckler.de, Tel.: 0211/7778-111 